Laser Chem. 1988, Vol. 9, pp. 147-154

(C) 1988 Harwood Academic Publishers GmbH

Photocopying permitted by license only

Reprints available directly from the Publisher

Printed in the United Kingdom

\title{
Analysis of the Dynamics of the Interaction of Ultraviolet Lasers with Organic Polymers
}

\author{
R. SRINIVASAN, EMILE SUTCLIFFE and BODIL BRAREN \\ IBM, T. J. Watson Research Center, Yorktown Heights, NY 10598
}

(Received January 15, 1988; in final form March 2, 1988)

The time-dependent treatment of the dynamics of the ablation of polymers that was proposed by Sutcliffe and Srinivasan has been used here to analyze the etching of several polymers by pulsed, ultraviolet laser radiation. For five polymers which span a wide range of absorptivities in the ultraviolet, the ablation threshold which is expressed in terms of the "useful" photon density in the ablation volume falls within a narrow range $( \pm 50 \%)$. The most intense absorbers show the most deviation from the average value. The flux threshold which defines the absorbed photon level above which the photons are useful in producing ablation is strongly influenced by the absorptivity of the polymer. The extension of these ideas to the ablation of tissue which contains only $20 \%$ polymer is also discussed.

KEY WORDS: Polymer ablation, UV laser etching, excimer lasers.

\section{INTRODUCTION}

Although five years have elapsed since the first report on the ablation and etching of polymers by pulsed, ultraviolet laser radiation was published, ${ }^{1-3}$ the chemical nature of the reactions that are involved in the process is far from clear. Fragmentation products such as atoms, diatomics, small molecules and small fragments of the polymer chain are all observed. ${ }^{4-8}$ Ablation is believed to be a volume explosion ${ }^{9}$ which would make the production of small molecules important. Experimental data which relate the etch depth/pulse to the fluence of 
the pulse have been analyzed by many workers. The first attempt to take the temporal width of the laser pulse into account was published by Keyes, Clarke and Eisner. ${ }^{10}$ The analysis of the dynamics that was proposed by Sutcliffe and Srinivasan ${ }^{11}$ also followed a time-dependent analysis of the process and assigned a large role to chromophores in the polymer chain which were doubly or multiply excited by the successive absorption of two or more photons. This is particularly true at wavelengths longer than $193 \mathrm{~nm}$ because decomposition from multiply excited electronic states is not only fast (on the time scale of molecular vibrations) but proceeds with high efficiency in terms of the number of centers that are excited. Most importantly, such reactions yield small molecules as products. The elementary steps which underlie this analysis will be discussed in detail in a later section.

The purpose of this study was to examine the etch behavior of a number of common polymers with a view to analyse the dynamics of their interaction with the laser at several UV wavelengths. It was of interest to see how the magnitudes of the flux threshold and the

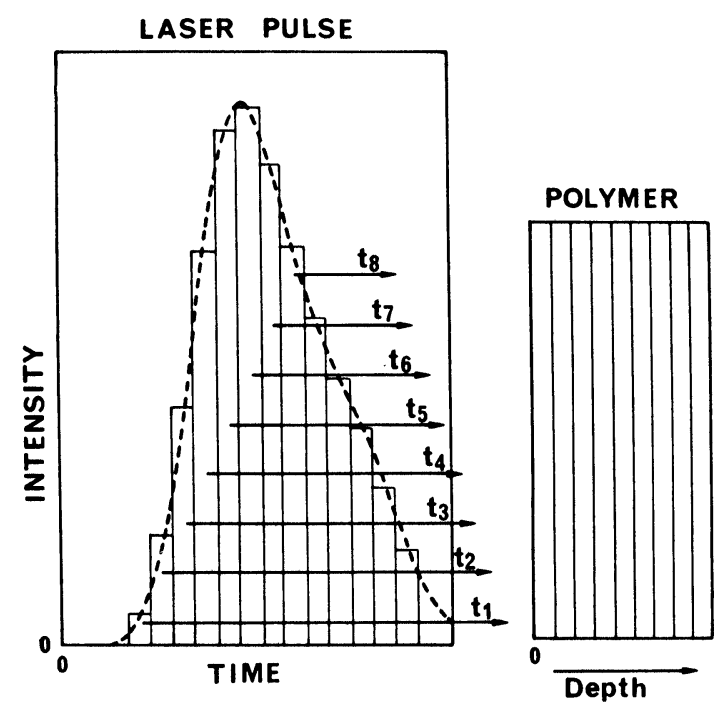

Figure 1 Conceptual view of interaction of laser pulse (left) with polymeric solid. The light pulse is sliced into many short pulses which are successively absorbed in hypothetical layers of the polymer. A layer will ablate when its effective concentration of absorbed quanta reaches the ablation threshold. 
ablation threshold of these various polymers relate to their structures and their UV absorption characteristics.

\section{EXPERIMENTAL}

Details of the experimental methods for the determination of etch curves for a given polymer at various excimer laser wavelengths have been described elsewhere. ${ }^{12}$ The widths (FWHM) of the pulses that were generated by the Lambda-Physik $201 \mathrm{E}$ lasers that were used ranged from $16 \mathrm{~ns}$ to $35 \mathrm{~ns}$. For the purpose of the present work, this variation was not found to be significant. The computer fitting of the results in order to evaluate the flux and ablation thresholds has been described. ${ }^{11}$

\section{THEORY}

The framework for the analysis of the dynamics is illustrated in Figures 1 and 2 . The volume of the polymer that is exposed to the radiation can

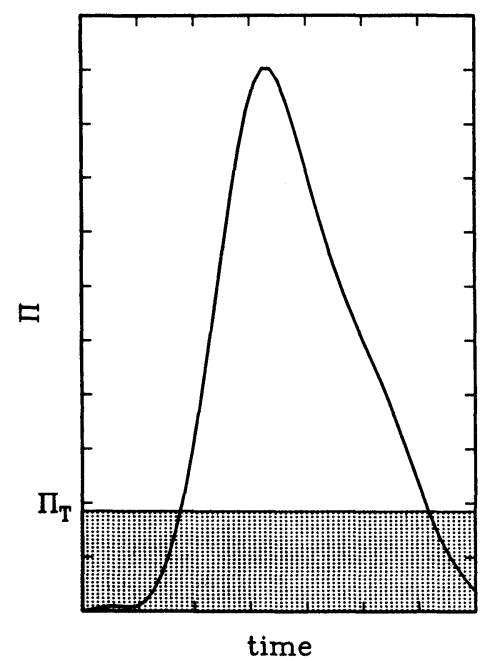

Figure 2 Schematic representation of the flux (П) of absorbed quanta as function of time in a slice of polymer. The plot indicates the concentration of absorbed quanta (= singly excited chromophores) in a given volume of polymer as a function of time as the laser pulse passes through the material. $\Pi_{T}$ represents the flux threshold (in concentration units) which must be exceeded before the relaxation process can be overcome. 
be conceptually viewed as being made up of several thousand elements which are individually only a fraction of a micron thick. When a pulse of photons sweeps through one such element, the concentration of absorbed photons (each of which would presumably lead to electronic excitation of a single chromophore) shows a time profile which mirrors the shape of the pulse itself. Since the decay of the electronically excited chromophores in the polymer chain is also time-dependent, a flux threshold, $\Pi_{T}$ can be defined which must be exceeded before ablation will become possible. The ablation threshold, $\rho_{T}$ is the concentration of "useful" photons, i.e., absorbed photons above $\Pi_{T}$ which must be reached before ablation will occur. This concept can be mathematically stated as in Eq. 1.

$$
\rho(t, x)=\int_{0}^{t}\left[\Pi\left(t^{\prime}, x\right)-\Pi_{T}\right] d t^{\prime}
$$

The key experimental evidence which showed the temporal dependence of the ablation was the photo-acoustic study on polymer ablation ${ }^{13}$ in which it was found that ablation began within a few nanoseconds of the onset of a pulse and came to an end along with the pulse.

Except in the far-UV, the quantum yield for the decomposition of organic molecules in the condensed phase with ultraviolet photons is invariably small $(<0.01)$ when electronic excitation is only to the first singlet or triplet states. ${ }^{14}$ It is therefore considered unlikely that ablation results from photochemical decomposition from singly excited chromophores. On the other hand when a chromophore is multiply-excited by the sequential absorption of two or more more photons, the total excitation energy which will exceed $8 \mathrm{eV}$ (at the very least) will be sufficient to cause multiple fragmentation and the production of small molecules. Evidence has been offered elsewhere ${ }^{5,6,15}$ that products of such a decomposition process are present in the ablation plume and show a large increase in concentration at fluences that exceed the threshold for ablation.

It is necessary to explain how Eq. 1 which is concerned only with the population of the first electronically excited state can deal with the dynamics of the laser ablation process. This is best discussed in terms of the four elementary chemical reactions, 2-5. 


$$
\begin{aligned}
& \mathbf{M}+\mathrm{h} v \longrightarrow \mathrm{M}^{*} \\
& \mathbf{M}^{*} \longrightarrow \mathrm{M} \\
& \mathrm{M}^{*}+\mathrm{h} v \longrightarrow \mathrm{M}^{* *} \\
& \mathrm{M}^{* *} \longrightarrow \text { Products }
\end{aligned}
$$

Here $M$ is a chromphore in a polymer chain and each asterisk denotes electronic excitation by one UV photon, and the $\alpha$ 's are absorptivities. From the stationary state condition,

$$
\text { Rate of decomposition }=\frac{I^{2} \alpha \alpha^{*}}{k_{3}+I \alpha^{*}}
$$

where $I$ is the incident photon flux. When and only when $I \alpha^{*} \gg k_{3}$, this expression reduces to $I \alpha$ so that the ablation rate can be determined simply by the excitation to the first upper state. The time-dependent analysis that is used will therefore be most suitable when $k_{3}$ is small (i.e. unimolecular deactivation processes are slow) which would be the case when a polymer is a relatively weak absorber and has a long radiative lifetime.

\section{RESULTS}

For polymers such as polyethylene and polypropylene which are weak absorbers even at $193 \mathrm{~nm}$, the process of fitting the experimental data to an etch curve can be carried out satisfactorily (Figure 3 and Figure 4)

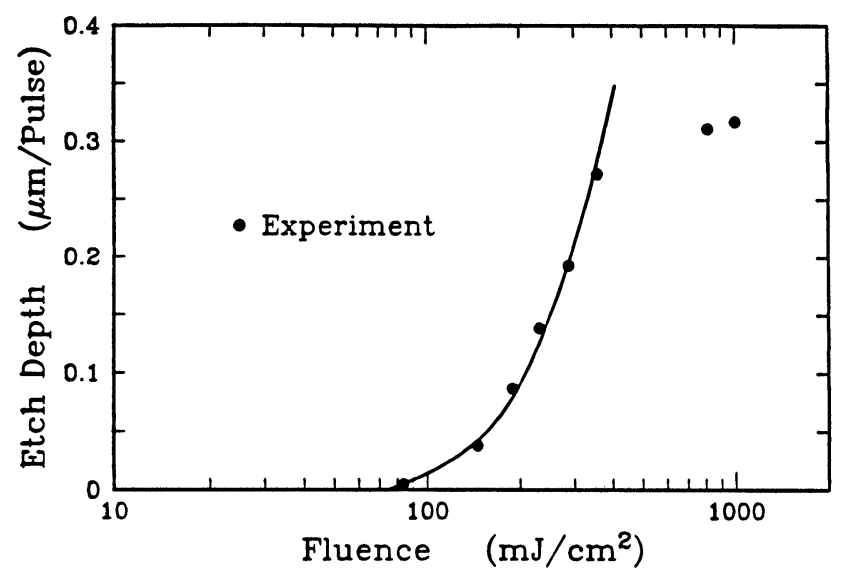

Figure 3 Fit of experimental data on the ablation of polyethylene to Eq. 1. 


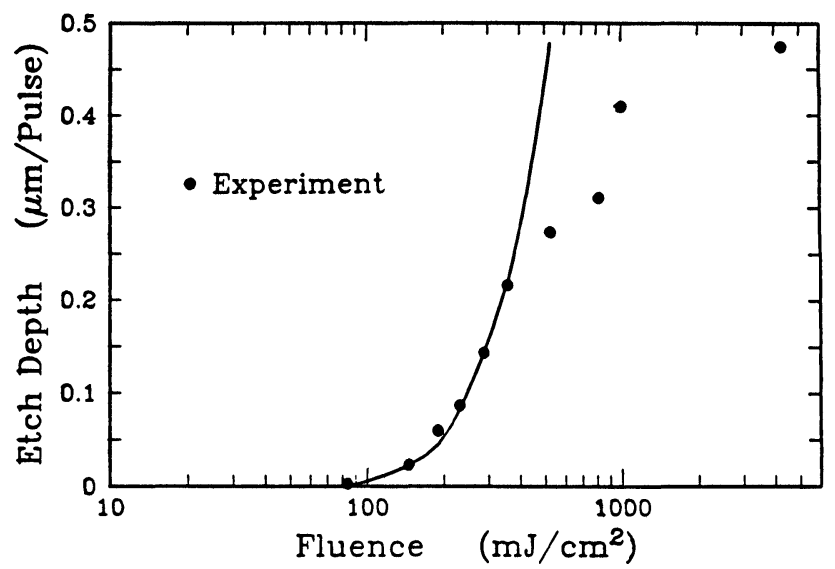

Figure 4 Fit of experimental data on the ablation of polypropylene to Eq. 1.

from the threshold fluence to the fluence value at which the etch depth starts to show a fall-off from the steadily rising curve. In contrast, for strongly absorbing polymers such as polystyrene (Figure 5) and polyimide, ${ }^{11}$ the etch curve can be fitted well only over a short fluence range. This behavior is now believed to be the result of the filtering action of the ablated material on the tail end of the pulse. ${ }^{16}$ This effect is not different from the behavior of weakly absorbing polymers in the fall-off region that was mentioned above.

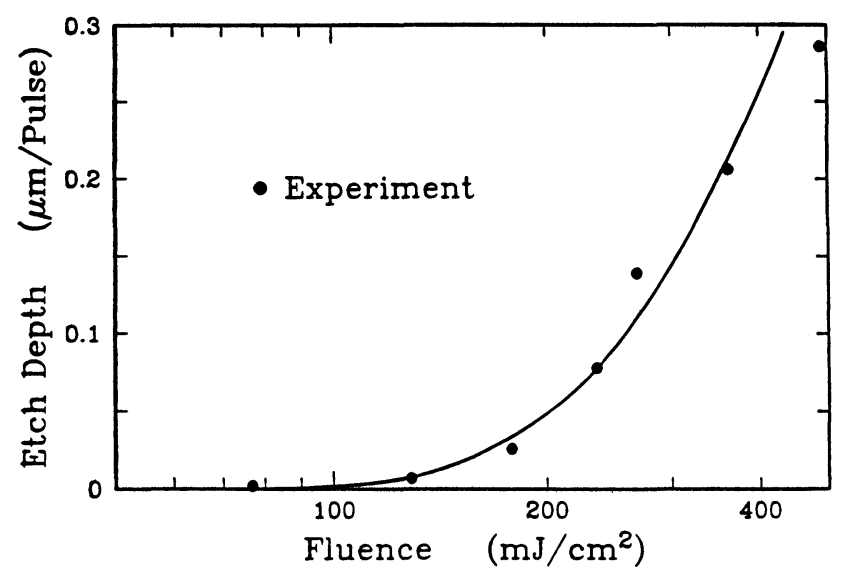

Figure 5 Fit of experimental data on the ablation of polystyrene to Eq. 1. 
The values of $\Pi_{T}$ and $\rho_{T}$ that were compiled are listed in Table I. The uncertainties cited in this table reflect the precision in the fitting process. The experimental uncertainties far exceed these values. Therefore, only the trends in the experimental values (and not the absolute values) should be considered to be significant.

For the five synthetic polymers, the value of $\Pi_{T}$ fits an average of $4.4 \times 10^{21}( \pm 50 \%)$ photons $/ \mathrm{cm}^{3}$. It suggests that when a critical number of chromophores are excited in a given volume, that material will ablate. Since the photon wavelength in the ultraviolet region is not constant in the results that are reported, the key feature must be the number of centers that react and not the total energy that is put in. This is consistent with the idea of a volume explosion in which the number of fragments that are produced in a given volume in unit time would determine the rise in pressure. The wavelength of the photon enters this picture mainly by way of the absorptivity of the polymer at that wavelength and secondarily in the fraction of the absorbed radiation that falls below the flux threshold and goes to heat the irradiated volume. ${ }^{11}$

The threshold values for the cornea which are also listed in Table I indicate that the ablation threshold is constant $( \pm 50 \%)$ only for solid polymers. Corneal tissue consists of about $80 \%$ of water. ${ }^{17}$ It should take very little energy to expel these water molecules which have been shown to leave the tissue as liquid water. ${ }^{18,19}$ Therefore, the ablation

Table I Flux threshold $\left(\Pi_{T}\right)$ and ablation threshold $\left(\rho_{T}\right)$ for several polymers and corneal tissue from fit of experimental data.

\begin{tabular}{lllll}
\hline & $\begin{array}{l}\Pi_{T} \text { (flux } \\
\text { threshold) } \\
10^{27} \text { phot./sec cm }\end{array}$ & $\begin{array}{l}\rho_{T} \text { (ablation } \\
\text { threshold) } \\
10^{21} \text { phot./cm }\end{array}$ & \multicolumn{2}{l}{ Fit parameters } \\
\cline { 4 - 5 } & & $\begin{array}{l}\text { Wavelength } \\
\mathrm{nm}\end{array}$ & $\begin{array}{l}\text { Absorptivity } \\
\mathrm{cm}^{-1}\end{array}$ \\
\hline Polyethylene & $2.3 \pm 0.1$ & $4.6 \pm 0.2$ & 193 & 640 \\
Polypropylene $^{3}$ & $3.0 \pm 0.1$ & $4.0 \pm 0.2$ & 193 & 566 \\
PMMA $^{\mathrm{a}}$ & $4.3 \pm 0.6$ & $4.1 \pm 0.4$ & 193 & 4600 \\
Polystyrene $_{\text {Polyimide }^{\mathrm{a}}}^{28 \pm 3}$ & $310 \pm 40$ & $6.4 \pm 0.9$ & 248 & 4100 \\
Cornea $^{\mathrm{b}}$ & $4.1 \pm 0.6$ & $2.8 \pm 1.9$ & 351 & $3 \times 10^{4}$ \\
\hline
\end{tabular}

\footnotetext{
${ }^{\text {a }}$ Ref. 11.

b Ref. 20.

c These values can be converted to $\mathrm{KJ} / \mathrm{cm}^{3}$ by dividing by $9.64 \times 10^{20}(193 \mathrm{~nm})$; $1.24 \times 10^{21}(248 \mathrm{~nm}) ; 1.75 \times 10^{21}(351 \mathrm{~nm})$.
} 
threshold needs to be $1 / 5$ th as large as for the polymers. The experimental value of $0.6 \times 10^{21}$ photons $/ \mathrm{cm}^{3}$ is close to the value of $0.9 \times 10^{21}$ photons $/ \mathrm{cm}^{3}$ which can be derived by multiplying the average value of $\rho_{T}$ for the solid polymers by 0.2 .

\section{References}

1. R. Srinivasan and V. Mayne-Banton, Appl. Phys. Lett. 41, 576 (1982).

2. R. Srinivasan and W. J. Leigh, J. Am. Chem. Soc. 104, 6784 (1982).

3. For a recent review, see R. Srinivasan, Science 234, 559 (1986).

4. M. W. Geis, J. N. Randall, T. F. Deutsch, N. N. Efremow, J. P. Donnelly and J. D. Woodhouse, J. Vac. Sci. Technol. B1 4, 1178 (1983).

5. G. M. Davis, M. C. Gower, C. Fotakis, T. Efthimiopoulos and P. Argyrakis, Appl. Phys. A 36, 27 (1985).

6. R. Srinivasan, B. Braren, D. E. Seeger and R. W. Dreyfus, Macromolecules 19, 916 (1986).

7. J. Brannon, J. R. Lankard, A. I. Baise, F. Burns and J. Kaufman, J. Appl. Phys. 58, 2036 (1985).

8. R. Larciprete and M. Stuke, Appl. Phys. B 42, 181 (1987).

9. B. J. Garrison and R. Srinivasan, Appl. Phys. Lett. 44, 849 (1984); J. Appl. Phys. 57, 2909 (1985).

10. T. Keyes, R. H. Clarke and J. M. Isner, J. Phys. Chem. 89, 4194 (1985).

11. E. Sutcliffe and R. Srinivasan, J. Appl. Phys. 60, 3315 (1986).

12. R. Srinivasan and B. Braren, J. Polymer Sci. 22, 2601 (1984).

13. P. E. Dyer and R. Srinivasan, Appl. Phys. Lett. 48, 445 (1986).

14. W. J. Leigh and R. Srinivasan, Acct. Chem. Res. 20, 107 (1987) and earlier references therein.

15. R. Srinivasan, B. Braren and R. W. Dreyfus, J. Appl. Phys. 61, 372 (1987).

16. R. Srinivasan and B. Braren, to be published.

17. D. M. Maurice, in "The eye," H. Dawson, Ed., Academic Press, New York (1969), Vol. 1, p. 489.

18. R. Srinivasan, P. E. Dyer and B. Braren, Lasers Surg. Med. 6, 514 (1987).

19. C. A. Puliafito, R. F. Steinert, T. F. Deutsch, F. Hillenkamp, E. J. Dehm and C. M. Adler, Ophthalmology 92, 741 (1985).

20. R. R. Krueger and S. L. Trokel, Arch. Ophthalmol. 103, 1741 (1985). 